\title{
Multiplex TaqMan qPCR assay for specific identification of encapsulated Trichinella species prevalent in North America
}

\author{
Marcos de Almeida ${ }^{1 /}{ }^{+}$, Henry Bishop' ${ }^{1}$ Fernanda S Nascimento', Blaine Mathison'1, \\ Richard S Bradbury', Alexandre da Silva ${ }^{2}$ \\ ${ }^{1}$ Centers for Disease Control and Prevention, Division of Parasitic Diseases and Malaria, Center for Global Health, Atlanta, GA, USA \\ ${ }^{2}$ US Food and Drug Administration Center for Food Safety and Applied Nutrition, Office of Applied Nutrition and Safety Assessment, \\ Laurel, MD, USA
}

BACKGROUND Human trichinellosis is a foodborne parasitic zoonotic disease caused by ingestion of raw or undercooked meat infected with nematode larvae of the genus Trichinella. In the USA, sporadic cases and outbreaks caused by the consumption of wild game meat infected with Trichinella have been reported. The current methods for diagnosis such as serology and microscopy are not specific, may result in false negative results, and cannot differentiate encapsulated Trichinella larvae to species level. The molecular protocols currently available for the differentiation of all encapsulate Trichinella species prevalent in North America have some limitations such as the inability to identify and resolve the presence of several Trichinella species in a single test.

OBJECTIVES/METHODS In this study we developed and evaluated a multiplex TaqMan quantitative real-time polymerase chain reaction (qPCR) assay, which can simultaneously detect, identify and differentiate all species of encapsulated Trichinella occurring in North America i.e., T. nativa, T. spiralis, T. murrelli and Trichinella T6, even in cases of multiple infection in a single sample. We investigated two human biopsies and 35 wild animal meat samples considered as having a high likelihood of harboring Trichinella larvae obtained from the United States during 2009-2017.

FINDINGS Using the multiplex assay describe here, $22(59 \%)$ samples that tested positive contained Trichinella spp., were identified as: $T$. nativa $(\mathrm{n}=7$, including a human biopsy), T. spiralis $(\mathrm{n}=9$, including a human biopsy), T. murrelli $(\mathrm{n}=3)$, Trichinella T6 $(\mathrm{n}=1)$. Results also included two rare mixed infection cases in bears, a T. nativa/T. spiralis from Alaska and a T. spiralis/Trichinella T6 from California. The species identifications were confirmed using a conventional PCR targeting the rRNA ITS1-ITS2 region, followed by DNA sequencing analysis. The estimated limit of detection (LOD) was approximately seven larvae per gram of meat.

MAIN CONCLUSIONS Differentiation of Trichinella spp. is needed to improve efforts on identification of case, optimize food safety control and better understand the geographic distribution of Trichinella species. The Trichinella qPCR multiplex proved to be a robust, easy to perform assay and is presented as an improved technique for identification of all known encapsulated species occurring in North America continent.

Key words: Trichinella species - clinical/environmental infection - microscopy - real-time PCR - conventional PCR and sequencing specific identification

Trichinellosis is a foodborne parasitic zoonotic disease caused by nematodes of the genus Trichinella. The disease represents both a public health hazard and a food safety problem in many parts of the world..$^{(1,2,3)}$

Human infection is acquired by ingestion of raw or undercooked infected meat of wild and game animals, and domestic (i.e., home-raised) or commercial pork meat. Although improvements in animal husbandry practices and implementation of federal programs to control the quality of processed meat products have contributed to the reduction in the number of reported cases of human trichinellosis since 1990's in the United States, occasional outbreaks and sporadic cases associated with consumption of wild game meat continue to occur. ${ }^{(4,5,6,7)}$

doi: 10.1590/0074-02760180305

Financial support: CDC/CGH/DPDM Atlanta, GA.

+ Corresponding author: bnz0@cdc.gov

Received 26 June 2018

Accepted 18 September 2018
Trichinellosis is a low prevalence disease in the United States and human infections may present with symptoms commonly observed in other diseases, ${ }^{(8)}$ contributing to delays in diagnosis of individual cases and identification of outbreaks. Standard laboratory methods used for diagnosis such as serology (the currently accepted gold standard diagnostic method) and microscopy may produce false negative results depending on the stage of disease and the larval load in the sample. ${ }^{(1,8)}$ In addition, using microscopy analysis, Trichinella species can only be differentiated to whether they are encapsulated or not, which does not allow precise identification of all species classified to date. As parasite capacity to survive freezing temperatures varies by species, ${ }^{(9,10)}$ the ability to differentiate species has important epidemiological and food surveillance implications. Therefore, efficient laboratory methods are needed to improve identification of clinical and environmental cases and better understand disease dissemination and the geographic distribution of the various Trichinella species in the North America.

Molecular procedures have the advantage of being both sensitive and specific for the identification of Trichinella parasites and have been applied to several epide- 
miological and taxonomic studies. As traditional testing approaches cannot distinguish Trichinella to species level, polymerase chain reaction (PCR)-based methods, which typically have higher sensitivity compared to microscopy, are useful for the identification of a variety of Trichinella species, ${ }^{(11,12)}$ including those causing human infection, ${ }^{(3,5,6,13)}$ and mixed infections in cases reported from focal areas of Europe and South Africa. ${ }^{(14,15,16)}$

Quantitative real-time PCR (qPCR) platforms are advantageous in comparison to conventional PCR tests, as they are more accurate, reduce the risk of contamination of the laboratory environment, and are less time consuming and labor intensive. The qPCR platforms have been increasingly applied to the detection and specific identification of Trichinella species. ${ }^{(17,18)}$ However, the protocols currently available cannot identify and resolve the presence of several Trichinella species in a single assay.

To facilitate the identification of clinical and environmental trichinellosis cases, we developed a multiplex TaqMan qPCR assay targeting the ribosomal RNA ITS1 region (rRNA-ITS1), for the specific identification of four encapsulated Trichinella species known to occur in North America. We analyzed meat samples implicated as the source of outbreaks or sporadic cases of human trichinellosis, comparing the multiplex TaqMan qPCR assay with microscopy results.

\section{MATERIALS AND METHODS}

Sample selection - Two clinical samples and 35 wild game meat samples submitted to the CDC's Parasitic Diseases Reference Diagnostic Laboratory between 2009-2017 were analyzed. The meat samples were obtained from animals hunted in several US states: 12 of walrus (Alaska), 11 samples of bear (Alaska $n=3$, California $\mathrm{n}=7$, Wisconsin $\mathrm{n}=1$ ), five of boar (Minnesota $\mathrm{n}$ $=2$, Missouri, California and Washington $\mathrm{n}=1$, respectively), four of deer (California $\mathrm{n}=3$ and Missouri $\mathrm{n}=$ 1), one of mountain lion (Idaho), one boar/deer mixed sausage (Missouri) and one deer/bear mixed sausage (California). All samples were considered to be potential sources for either outbreaks or individual cases of trichinellosis in humans. In addition, T. nativa (code ISS70 and ISS1751), T. spiralis (code ISS3 and ISS328), T. murrelli (code ISS346 and ISS415), Trichinella T6 (code ISS34 and ISS40) and T. pseudospiralis (code ISS13, ISS470 and ISS4134) larvae isolates were provided by Dr. Edoardo Pozio, Instituto Superiore di Sanita - European Union Reference Laboratory for Parasites for use as reference controls. Samples are listed in Table I.

Microscopic analysis - Initial analysis of the 37 samples was performed by compressing fragments of meat (roast and steak) of approximately $0.25 \mathrm{~g}$ in weight between two glass slides. These samples were examined by two independent expert analysts using light microscopy at 100x magnification to detect larvae of Trichinella spp. If no larvae were detected, a larger aliquot of the sample, determined by the total amount of the sample available, was partially digested using artificial gastric juice (aqueous solution of $0.5 \%$ pepsin and $0.7 \%$ hydrochloric acid), incubated at $37^{\circ} \mathrm{C}$ for a minimum of $4 \mathrm{~h}$, and examined.
(19) The microscopic analysis reproducibility was verified by reexamining a blinded panel of 12 new aliquots of these 37 samples. Larval loads for the determination of limit of detection (LOD) of the novel Trichinella multiplex qPCR were determined by digestion of approximately $0.25 \mathrm{~g}$ of three Trichinella positive meat samples, then counting the number of larvae within each sample.

DNA extraction - DNA was extracted from approximately $0.25 \mathrm{~g}$ cubes of meat and from an average of five larvae of each reference control isolates using DNeasy Blood \& Tissue Kit (Qiagen, Germantown, MD), following the manufacturer's instructions.

Oligonucleotide design and TaqMan real-time PCR optimization - Generic ribosomal RNA (rRNA) gene sequences of the different nematode species, including Trichinella spp. available in GenBank were aligned and used to design specific oligonucleotides to amplify fragments of approximately $3540 \mathrm{bp}$ encompassing the fulllength 18S-ITS1-5.8S-ITS2 rRNA of T. nativa, T. murrelli, T. spiralis and Trichinella T6. These DNA fragments were cloned using pCR2.1-TOPO vector (Invitrogen-Thermo Fisher, Waltham, MA) following the manufacturer directions and sequenced for reference (data not shown). The Trichinella multiplex qPCR assay was prepared using primers TCN-ITS1 2307F and TCN-ITS1 2411R, specific for the four known encapsulated Trichinella species occurring in North America. The primers were designed to amplify fragments of rRNA-ITS1 region ranging from 85 to $98 \mathrm{bp}$ depending on the species (nucleotides 2285 to 2370, based on T. nativa accession no. KP307962). Four species-specific probes TnatR, TsprlR, TmurR and T6R labeled with VIC, CY5, FAM and NED and using minor grove binding (MGB) quenchers (Applied BiosystemsThermo Fisher, Waltham, MA) were designed for differential detection of T. nativa, T. spiralis, T. murrelli, and Trichinella T6, respectively. Oligonucleotide sequences, nucleotide biding position (target) and GenBank references are listed in Table II.

The specificity of the Trichinella multiplex qPCR assay was evaluated by comparing results with an inhouse ITS1-ITS2 conventional PCR (Table II), followed by DNA sequencing analysis. These reactions were prepared with genus-level primers TCN-ITS1 2407F and TCN-28S 3511R designed to amplify fragments ranging between 1140-1167 bp depending on the species (nucleotides 2375-3519, T. nativa accession no. KP307962 as reference). In addition, internal primers TCN-ITS2 3020F TCN-ITS2 3020R were used for alternative amplification and for DNA sequencing analysis in cases where the amplification with ITS1-ITS2 PCR failed. Fig. 1 shows a diagram depicting the areas these PCR reactions targets within rRNA ITS1-5.8S-ITS2 sequence.

SYBR Green methods used for quality control and comparison - To assure the quality of the 37 DNA extracts and to minimize possible false negative results, a SYBR Green qPCR screening reaction were designed to amplify the animals DNA extracts using the generic primers 18SrRNA-F and 18SrRNA-R. In addition, another SYBR Green using the primers ESVF and 


\section{TABLE I}

List of samples, reference Trichinella spp. larvae isolates and nematode DNA tested by Trichinella quantitative real-time polymerase chain reaction (qPCR) multiplex assay and microscopy analysis. Species were identified using Trichinella qPCR multiplex assay and confirmed by DNA sequencing analysis of the Trichinella ribosomal RNA gene

\begin{tabular}{|c|c|c|c|c|}
\hline Sample ID & Host, origin, year & $\begin{array}{l}\text { Trichinella multiplex qPCR } \\
\text { assay (Dye/Ct value) }\end{array}$ & $\begin{array}{l}\text { Trichinella rRNA gene } \\
\text { sequencing result }\end{array}$ & $\begin{array}{l}\text { Microscopy result } \\
\text { for } 0.25 \mathrm{~g} \text { of sample }\end{array}$ \\
\hline SP\#1 & Human, MN, 2011 & T. spiralis (CY5 - Ct28) & T. spiralis & NEG \\
\hline SP\#2 & Human, AK, 2014 & T. nativa (VIC - Ct28) & T. nativa & POS \\
\hline $\mathrm{SP} \# 3$ & Bear, CA, 2006 & T. murelli (FAM - Ct36) & T. murelli & 2 larvae \\
\hline $\mathrm{SP} \# 4$ & Bear, CA, 2007 & T. murelli (FAM - Ct17) & T. murelli & POS \\
\hline $\mathrm{SP} \# 5$ & Bear, CA, 2009 & T. murelli (FAM - Ct25) & T. murelli & POS \\
\hline SP\#6 & Boar, MN, 2011 & T. spiralis (CY5 - Ct28) & T. spiralis & NEG \\
\hline SP\#7 & Boar, MN, 2011 & T. spiralis (CY5 - Ct26) & T. spiralis & NEG \\
\hline SP\#8 & Boar, WA, 2012 & NEG & NEG & NEG \\
\hline $\mathrm{SP} \# 9$ & Bear, CA, 2012 & NEG & NEG & NEG \\
\hline SP\#10 & Bear, AK, 2013 & $\begin{array}{l}\text { T. nativa }(\mathrm{VIC}-\mathrm{Ct} 24) \\
\text { T. spiralis }(\mathrm{CY} 5-\mathrm{Ct} 32)\end{array}$ & $\begin{array}{l}\text { T. nativa } \\
\text { T. spiralis }\end{array}$ & 15 larvae \\
\hline SP\#11 & Boar, MO, 2013 & T. spiralis (CY5 - Ct30) & T. spiralis & POS \\
\hline $\mathrm{SP} \# 12$ & Deer, MO, 2013 & NEG & NEG & NEG \\
\hline SP\#13 & Boar/Deer, MO, 2013 & T. spiralis (CY5 - Ct37) & T. spiralis & NEG \\
\hline SP\#14 & Mountain lion, ID, 2014 & Trichinella T6 (NED - Ct30) & Trichinella $\mathrm{T} 6$ & POS \\
\hline $\mathrm{SP} \# 15$ & Bear, CA, 2015 & T. spiralis (CY5 - Ct27) & T. spiralis & POS \\
\hline SP\#16 & Bear, CA, 2015 & T. spiralis (CY5 - Ct32) & T. spiralis & POS \\
\hline SP\#17 & Bear, CA, 2015 & T. spiralis (CY5 - Ct26) & T. spiralis & 21 larvae \\
\hline SP\#18 & Bear, AK, 2015 & T. nativa (VIC - Ct25) & T. nativa & POS \\
\hline $\mathrm{SP} \# 19$ & Bear, AK, 2016 & NEG & NEG & NEG \\
\hline SP\#20 & Bear, WI, 2016 & T. nativa (VIC - Ct37) & T. nativa & POS \\
\hline SP\#21 & Boar, CA, 2017 & T. spiralis (CY5 - Ct26) & T. spiralis & POS \\
\hline $\mathrm{SP} \# 22$ & Walrus, AK, 2017 & T. nativa (VIC - Ct36) & T. nativa & NEG \\
\hline $\mathrm{SP} \# 23$ & Walrus, AK, 2017 & T. nativa (VIC - Ct35) & T. nativa & NEG \\
\hline SP\#24 & Walrus, AK, 2017 & NEG & NEG & NEG \\
\hline $\mathrm{SP} \# 25$ & Walrus, AK, 2017 & NEG & NEG & NEG \\
\hline $\mathrm{SP} \# 26$ & Walrus, AK, 2017 & T. nativa (VIC - Ct26) & T. nativa & NEG \\
\hline SP\#27 & Walrus, AK, 2017 & NEG & NEG & NEG \\
\hline SP\#28 & Walrus, AK, 2017 & T. nativa (VIC - Ct26) & T. nativa & POS \\
\hline SP\#29 & Walrus, AK, 2017 & NEG & NEG & NEG \\
\hline $\mathrm{SP} \# 30$ & Walrus, AK, 2017 & NEG & NEG & NEG \\
\hline $\mathrm{SP} \# 31$ & Walrus, AK, 2017 & NEG & NEG & NEG \\
\hline $\mathrm{SP} \# 32$ & Walrus, AK, 2017 & NEG & NEG & NEG \\
\hline $\mathrm{SP} \# 33$ & Walrus, AK, 2017 & NEG & NEG & NEG \\
\hline SP\#34 & Dear, CA, 2017 & NEG & NEG & NEG \\
\hline $\mathrm{SP} \# 35$ & Dear, CA, 2017 & NEG & NEG & NEG \\
\hline $\mathrm{SP} \# 36$ & Dear, CA, 2017 & NEG & NEG & NEG \\
\hline $\mathrm{SP} \# 37$ & Dear/Bear, CA, 2017 & $\begin{array}{l}\text { T. spiralis }(\mathrm{CY} 5-\mathrm{Ct} 37) \\
\text { Trichinella } \mathrm{T} 6(\mathrm{NED}-\mathrm{Ct} 25)\end{array}$ & $\begin{array}{l}\text { T. spiralis } \\
\text { Trichinella } \mathrm{T} 6\end{array}$ & POS \\
\hline T. nativa $a^{a}$ & $\begin{array}{c}\text { Wolf, Russia, } 1987 \text { (ISS70) } \\
\text { Bear, Canada, } 2005 \text { (ISS1751) }\end{array}$ & VIC positive - (Ct24) & T. nativa & NA \\
\hline T. spiralis ${ }^{a}$ & $\begin{array}{l}\text { Pig, Poland, } 1960 \text { (ISS3) } \\
\text { Pig, Sweden, } 1994 \text { (ISS328) }\end{array}$ & CY5 positive - $(\mathrm{Ct} 23)$ & T. spiralis & NA \\
\hline T. murrelli ${ }^{a}$ & $\begin{array}{c}\text { Bear, USA, } 1994 \text { (ISS348) } \\
\text { Raccoon, USA, } 1989 \text { (ISS415) }\end{array}$ & FAM positive - $(\mathrm{Ct} 25)$ & T. murrelli & NA \\
\hline Trichinella $T 6^{a}$ & $\begin{array}{c}\text { Grizzly, USA, } 1983 \text { (ISS34) } \\
\text { Mountain lion, USA, } 1985 \text { (ISS40) }\end{array}$ & NED positive - (Ct28) & Trichinella T6 & NA \\
\hline T. pseudospiralis ${ }^{a}$ & $\begin{array}{c}\text { Raccoon, Russia, } 1972 \text { (ISS13) } \\
\text { Black vulture, USA, } 1995 \text { (ISS470) } \\
\text { Boar, Italy, (ISS4134) }\end{array}$ & NEG & NA & NA \\
\hline Human/Helminths ${ }^{b}$ & & NEG & NA & NA \\
\hline
\end{tabular}

$a$ : larvae isolates positive controls - Instituto Superiore di Sanita, European Union Reference Laboratory for Parasites; $b$ : Taenia solium, Paragonimus mexicanus, Trichuris sp., Halicephalobus sp., Anisakis simplex, Brugia malayi, Dirofilaria immitis, Onchocerca lupi, Onchocerca volvulus, and Wuchereria bancrofti; NA: not available; NEG: negative; POS: positive. 
TABLE II

Oligonucleotide sequences used for detection and identification of Trichinella nativa, T. spiralis, T. murrelli and Trichinella T6

\begin{tabular}{|c|c|c|c|c|}
\hline Name & Primer sequences $^{a}$ & Type of oligo - Reaction & nt Target ${ }^{b}$ & Reference $^{c}$ \\
\hline TCN-ITS1 2307F & GAG TGT GAC CAA AAT GAG AAA CC & Primer - TaqMan & 2285-2307 (ITS1) & 7962 \\
\hline TCN-ITS1 2411R & CAA ACC TAT TGA AAC CCA AGC AC & Primer - TaqMan & $2357-2370$ (ITS1) & KP307962 \\
\hline TnatR & VIC-AAC ACA AAA AAT AAA C-MGBNFQ & Probe - TaqMan & $2527-2342$ (ITS1) & KP307962 \\
\hline TsprlR & FAM-AGC ACA TTA CAC TGC ACT-MGBFNQ & Probe - TaqMan & $2338-2355$ (ITS1) & KC006422 \\
\hline TmurR & FAM-AAC ACA CTG AGC ACT ACA-MGBNFQ & Probe - TaqMan & $2335-2352$ (ITS1) & KC006408 \\
\hline TT6R & NED-CAA ACA CTA AAA TAA-MGBNFQ & Probe - TaqMan & 2322-2336 (ITS1) & KP307967 \\
\hline TCN-ITS1 2407F & GGT CAA CCG CCA CGT CCA ATC & Primer - PCR, sequencing & 2375-2395 (ITS1) & KP307962 \\
\hline TCN-28S 3511R & CTC GCC GCT ACT TGG AGA ATT CG & Primer-PCR, sequencing & 2497-3519 (ITS2) & KP307962 \\
\hline TCN-ITS2 3020F & TGT CGA CGT TGC AGT GTG TG & Primer - PCR, sequencing & 2957-2976 (ITS2) & KP307962 \\
\hline TCN-ITS2 3020R & CAC ACA CTG CAA CGT CGA CA & Primer - PCR, sequencing & 2957-2976 (ITS2) & KP307962 \\
\hline 18SrRNA-F & TAT GCG ACT ACC ATG GTG ATA AC & Primer - SYBR Green qPCR & $349-371(18 \mathrm{~S})$ & NA \\
\hline 18SrRNA-R & CTG CCT TCC TTG GAT GTG GTA & Primer - SYBR Green qPCR & $423-443(18 \mathrm{~S})$ & NA \\
\hline ESVF & GTT CCA TGT GAA CAG CAG T & Primer - SYBR Green qPCR & 1srDNA & (20) \\
\hline ESVR & CGA AAA CAT ACG ACA ACT GC & Primer - SYBR Green qPCR & 1srDNA & (20) \\
\hline
\end{tabular}

$a$ : primers are in 5'-3 'orientation; $b$ : nucleotide binding position; $c$ : GenBank accession references.

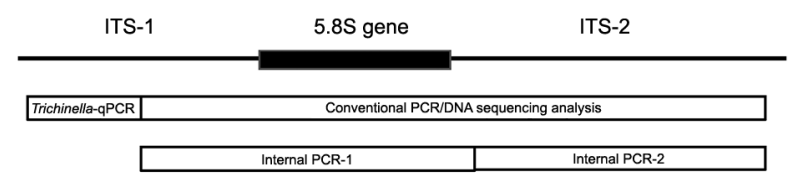

Fig. 1: polymerase chain reaction (PCR) targets at rRNA-ITS region. Trichinella quantitative real-time (qPCR) corresponds to nucleotides 2285 (TCN-ITS1 2307F ) to 2370 (TCN-ITS1 2411R). Conventional PCR/DNA sequencing analysis corresponds to nucleotides 2375 (TCN-ITS1 2407F) to 3519 (TCN-28S 3511R). Internal PCR 1 corresponds to nucleotides 2375 (TCN-ITS1 2407F) to 3006 (TCNITS2 3020R) and Internal PCR 2 corresponds to nucleotides 3006 (TCN-ITS2 302F) to 3519 (TCN-28S 3511R). T. nativa accession no. KP307962 was used as a reference.

ESVR, ${ }^{(20)}$ originally described to amplify $T$. nativa, $T$. spiralis, T. britovi, T. pseudospiralis, T. nelsoni, Trichinella T5 and Trichinella T6, were used to confirm the results on samples tested negative by the Trichinella spp. multiplex TaqMan qPCR.

The optimal annealing temperatures for qPCR primers and probes were determined using a $2^{\circ} \mathrm{C}$ gradient ranging from $56-64^{\circ} \mathrm{C}$. The optimal probe concentration was determined using a $50 \mathrm{nM}$ gradient ranging from 50-500 nM. Two independent reaction sets of each gradient were prepared in triplicate to assure the quality of results. The annealing temperature of the conventional PCR was empirically determined based on primer nucleotide sequences.

The specificity of the multiplex qPCR assay was determined by comparison to 20 human and multiple helminth DNA extracts identified in our laboratory in other investigations (these being those of Taenia solium, Paragonimus mexicanus, Trichuris sp., Halicephalobus gingivalis. Anisakis simplex, Brugia malayi, Dirofilaria immitis, Onchocerca lupi, Onchocerca volvulus and Wuchereria bancrofti).
The LOD of the Trichinella qPCR assay was demonstrated on the basis of the number of larvae (larvae per gram, lpg) determined by microscopy in three of the samples meat (SP\#3, SP\#10 and SP\#17). DNA was extracted from the $0.25 \mathrm{~g}$ of meat portions using the protocol described above and used in Trichinella qPCR reactions were prepared using a 10-fold DNA dilution gradient ranging from $10^{-1}$ to $10^{-6}$. LOD was calculated based on results of a triplicate set of reactions. In addition, another set of reactions prepared using dilutions of DNA extracts from larvae reference controls, were used to determinate a second parameter of LOD (data not shown).

qPCR reactions - The TaqMan reaction mixtures were prepared with 1-3 $\mu \mathrm{L}$ of DNA, $250 \mathrm{nM}$ each primer, $150 \mathrm{nM}$ of each probe, $12.5 \mu \mathrm{L}$ of TaqMan ${ }^{\circledR}$ Universal PCR Master Mix (Applied Biosystems - Thermo Fisher, Waltham, MA) and sterile water to adjust the volume to 25 $\mu \mathrm{L}$. The reactions were performed on an ABI 7500 realtime PCR system (Applied Biosystems - Thermo Fisher, Waltham, MA) using the following cycling parameters: $50^{\circ} \mathrm{C}$ for $2 \mathrm{~min}, 95^{\circ} \mathrm{C}$ for $10 \mathrm{~min}$, followed by 40 cycles of $95^{\circ} \mathrm{C}$ for $15 \mathrm{~s}$ and $60^{\circ} \mathrm{C}$ for $1 \mathrm{~min}$. Fluorescence data were collected at the end of each $60^{\circ} \mathrm{C}$ plateau.

The reaction mixtures for the two SYBR Green assays were prepared with $3 \mu \mathrm{L}$ of DNA, $250 \mathrm{nM}$ of each primer, 12.5 $\mu \mathrm{L}$ of QuantiTect SYBR Green PCR master mix (Qiagen, Germantown, MD) and sterile water to adjust the volume to $25 \mu \mathrm{L}$. The assays were run side-by-side on ABI 7500 real-time PCR system (Applied Biosystems - Thermo Fisher, Waltham, MA) using the following cycling parameters: $50^{\circ} \mathrm{C}$ for $2 \mathrm{~min}, 95^{\circ} \mathrm{C}$ for $15 \mathrm{~min}$, followed by 40 cycles of $95^{\circ} \mathrm{C}$ for $15 \mathrm{~s}$ and $60^{\circ} \mathrm{C}$ for $1 \mathrm{~min}$. Fluorescence data were collected at the end of each $60^{\circ} \mathrm{C}$ plateau.

Conventional PCR and DNA sequencing analysis Reactions were prepared with $3 \mu \mathrm{L}$ of DNA, $250 \mathrm{nM}$ of each primer and $45 \mu \mathrm{L}$ of Platinum Blue PCR SuperMix 
mixture (Invitrogen - Thermo Fisher, Waltham, MA) in a total volume of $50 \mu \mathrm{L}$. PCR was performed in a GeneAmp PCR System 9700 thermocycler (Applied Biosystems - Thermo Fisher, Waltham, MA). Reactions were performed using the following cycle structure: $95^{\circ} \mathrm{C}$ for 2 min, followed by 40 cycles of $95^{\circ} \mathrm{C}$ for $30 \mathrm{~s}, 60^{\circ} \mathrm{C}$ for 30 $\mathrm{s}$, and $72^{\circ} \mathrm{C}$ for $1 \mathrm{~min}$, with a final extension of $72^{\circ} \mathrm{C}$ for $5 \mathrm{~min}$. The amplicons were resolved in a $1.5 \%$ agarose gel, purified with StrataPrep PCR Purification Kit (Stratagene, San Diego, CA) and sequenced using BigDye version 3.1 chemistry (Applied Biosystems - Thermo Fisher, Waltham, MA) with primers TCN-ITS2 3020F and TCN-ITS2 3020R in addition to those used for PCR amplification (TCN-ITS1 2407F and TCN-28S 3511R). The sequencing reaction mixtures were purified through Sephadex Multi-Screen-HV plates (Millipore) and analyzed on an ABI Prism 3100 sequencer, with data collection software, version 2.0, and DNA Sequencing Analysis Software, version 5.1 (Applied Biosystems - Thermo Fisher, Waltham, MA). The sequences were assembled, edited, and aligned in DNA STAR SeqMan v. 14.0.0 (88) 422 (DNASTAR Inc., Madison, WI) software.

For specific identification of parasites in the specimens containing more than one species, DNA fragments amplified using primers TCN-ITS1 2407F/ TCN-ITS2 3020R and TCN-ITS2 3020F/ TCN-28S 3511R were used to prepare libraries using ThruPlex-FD Prep Kit (Rubicon Genomics, Ann Arbor, MI).

Amplicon library were prepared using 20 ng of input DNA and 10 cycles of amplification; purification was performed as per manufacturer's instructions. The libraries were quantified using a Quibit fluorometer 2.0 with HS reagent (Invitrogen -Thermo Fisher, Waltham, MA), NEBNext Library Quantification kit for Illumina (New England Biolabs, Ipswich, MA) and the library size was assessed using Tape Station 2200 gDNA Screen Tape (Agilent, Santa Clara, CA). The barcoded libraries were diluted to $4 \mathrm{nM}$ pooled and, $15 \mathrm{pM}$ loaded in a MiSeq Nano Kit 2x250 (Illumina, San Diego, CA). MiSeq reads were analyzed using MAFFT multiple alignment available in Geneious V.9 GeniousR9 - (9.1.5) software.

Ethics - Clinical samples were used in accordance with a CDC human subject-approved protocol - IRB\# 5756.

\section{RESULTS}

Reproducibility of microscopy analysis - Encysted Trichinella larvae were identified in 15 of the 37 samples $(40.5 \%)$ by microscopic analysis, including one of the two human biopsy specimens (Table I). New aliquots were prepared from 12 of the 37 samples for the reproducibility exercise (i.e., SP\#1, SP\#3 SP\#7, SP\#8, SP\#9, SP\#10, SP\#11, SP\#13, SP\#14, SP\#16, SP\#17 and SP\#29). Eight samples that were positive on first examination were also positive when re-examined. Samples SP\#11 and SP\#14, which were positive upon the first examination by microscopy, were negative when re-examined; Samples SP\#8 and $\mathrm{SP} \# 9$ were negative by both examinations.

Larval loads in samples SP\#3, SP\#10 and SP\#17 were determined as 8, 60 and 80 larvae/g (lpg) of meat, respectively (Fig. 2). DNA extracts from these naturally infected samples were used for the demonstration of the Trichinella qPCR multiplex assay LOD as described below.

Quality control, optimization and evaluation of LOD of Multiplex TaqMan qPCR reactions - All 10 Trichinella larvae DNA control extracts diluted at 1/50 were successfully amplified using this assay. The rRNA gene fulllength used as reference sequences on oligonucleotides design were submitted to GenBank database under accession\# KC006408-KC006421 (T. murrelli), KC006422KC006433 (T. spiralis), KP307962-KP307966 (T. nativa) and KP307967-KP307971 (Trichinella T6). The amplification of all 37 DNA extracts using the generic $18 \mathrm{Sr}-$ RNA SYBR Green qPCR demonstrated the integrity and absence of inhibitors in these DNA samples.

\begin{tabular}{|c|c|}
\hline Dilutions (SP\#10 - VIC) & Ct values \\
\hline undiluted & 26.31 \\
\hline $1 / 10$ & 29.61 \\
\hline $1 / 100$ & 32.20 \\
\hline $1 / 1000$ & 34.11 \\
\hline $1 / 10000$ & 36.70 \\
\hline Dilutions (SP\#10 - CY5) & Ct values \\
\hline undiluted & 31.75 \\
\hline $1 / 10$ & 34.24 \\
\hline $1 / 100$ & 36.84 \\
\hline $1 / 1000$ & 36.88 \\
\hline & \\
\hline Dilutions (SP\#17 - CY5) & Ct values \\
\hline undiluted & 26.65 \\
\hline $1 / 10$ & 32.61 \\
\hline $1 / 100$ & 36.02 \\
\hline $1 / 1000$ & 37.04 \\
\hline & \\
\hline & \\
\hline
\end{tabular}

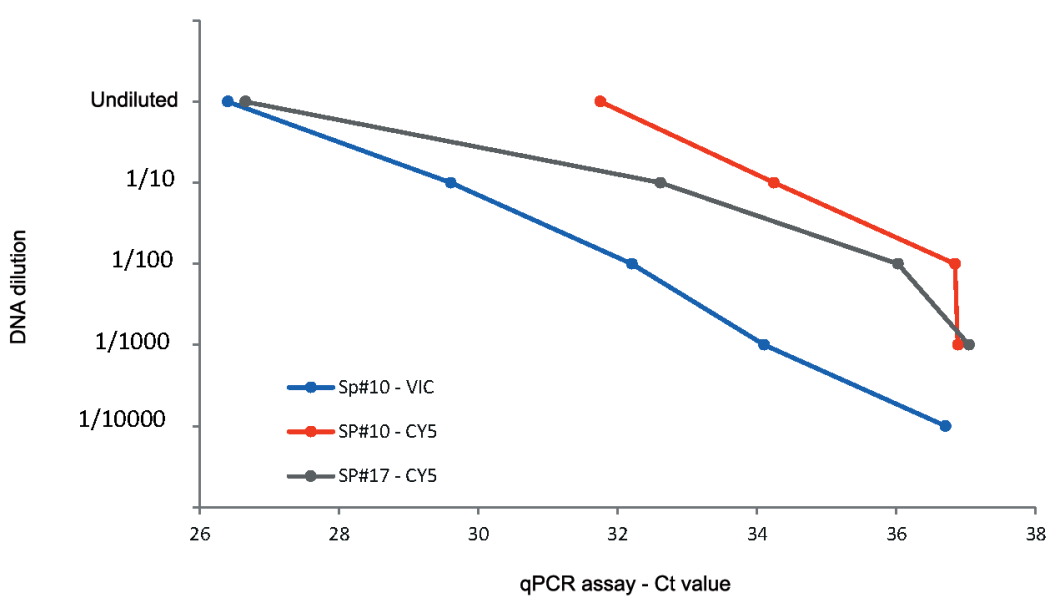

Fig. 2: Trichinella multiplex quantitative real-time polymerase chain reaction (qPCR) assay limit of detection (LOD). SP\#10 (T. spiralis and T. nativa mixed infection) and SP\#17 (T. spiralis only) were tested in triplicates of 10-fold series dilutions. The Ct values on the plots are the average of three runs of each sample. 
The optimal Trichinella multiplex qPCR assay annealing temperature and probe concentration were determined by gradient analysis to be between $58-62^{\circ} \mathrm{C}$ with between 100 to $200 \mathrm{nM}$ of probe per reaction. Based on these results, the optimal qPCR conditions were determined to be an annealing temperature of $60^{\circ} \mathrm{C}$ and $150 \mathrm{nM}$ of probe per reaction. The capacity of the assay to correctly identify and differentiate Trichinella species was determined using a combination of all probes in individual reactions containing T. nativa, T. spiralis, T. murrelli, Trichinella T6 and T. pseudospiralis DNA controls. With exception of T. pseudospiralis, we were able to detect and identify the species investigated (Table I, Fig. 3). DNA aliquots from human and the other helminth species were not amplified by the qPCR assay.

Using DNA extracts of samples SP\#3, SP\#10 and SP\#17, the LOD calculated for the Trichinella multiplex qPCR assay was $7 \times 10^{-3} \mathrm{lpg}$ of infected meat. T. murrel$l i$ was identified in an undiluted DNA aliquot of SP\#3 (FAM - Ct 37). In SP\#17, T. spiralis was detected in an undiluted aliquot $(\mathrm{Ct} 26)$ and in $1 / 10(\mathrm{Ct} \mathrm{30}), 1 / 100(\mathrm{Ct}$ 33) and $1 / 1000$ (Ct 37) dilutions. A mixed infection with both T. nativa and T. spiralis was detected in SP\#10, T. nativa was detected in an undiluted aliquot ( $\mathrm{Ct} 24)$, and in 1/10 (Ct 28), 1/100 (Ct 35) and 1/1000 (Ct 37) dilutions and $T$. spiralis was detected in an undiluted DNA aliquot only (Ct 37). We also ran Trichinella multiplex qPCR gradient reactions using T. nativa, T. spiralis, T. murrelli, Trichinella T6 DNA controls, these PCR preparations showed reactivity with DNA dilutions equivalent to < $10^{-15} \mathrm{~g} / \mu \mathrm{L}$ (data not shown).

A
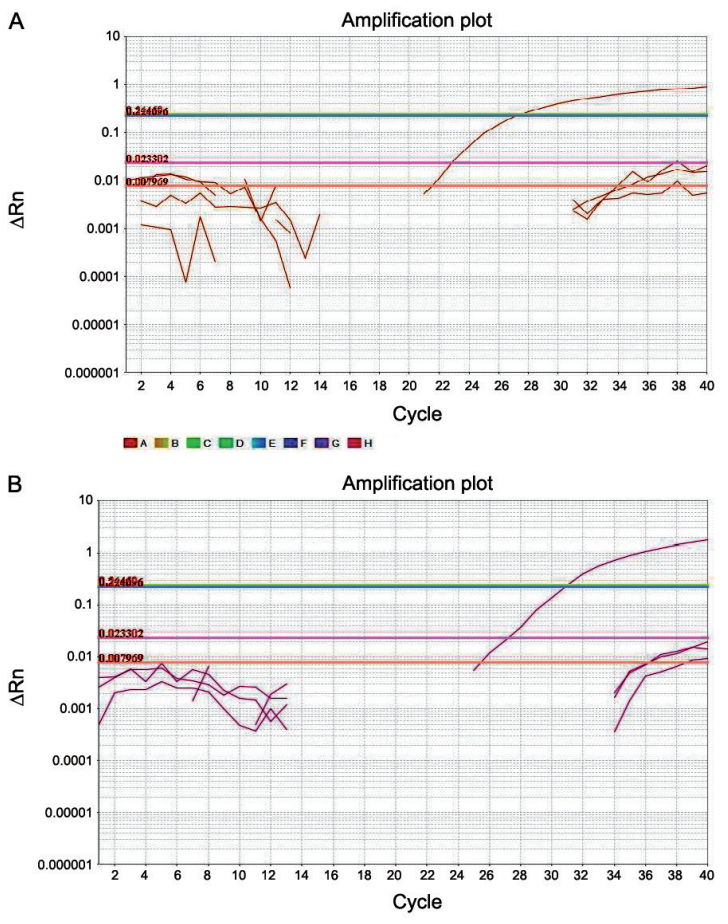

Trichinella spp. identification in clinical and meat samples. The qPCR multiplex assay detected Trichinella spp. in 22 out of 37 samples $(59 \%$, which included two human biopsies) with a total of 15 negative samples. The assay showed improved detection efficiency compared to conventional microscopic analysis since the 22 Trichinella spp. positive samples included the 15 samples positive by microscopy and seven other samples (SP\#1, SP\#6, SP\#7, SP\#13, SP\#22, SP\#23 and SP\#26) that were negative by microscopic analysis. Results on the 22 cases infected with Trichinella spp. and the 15 negative samples were confirmed in samples investigated by the ESV/ESVR SYBR Green qPCR reactions.

Twenty singly infected samples were identified as; $T$. nativa ( $\mathrm{n}=7$, including one clinical sample), T. spiralis $(\mathrm{n}=9$, including one clinical sample), T. murrelli $(\mathrm{n}=3)$ and Trichinella T6 $(\mathrm{n}=1)$. The method also efficiently identified two cases with mixed infection. Meat sample SP\#10 (bear - AK) contained a mixed infection of T. nativa (Ct 24)/T. spiralis (Ct 32) and sausage sample SP\#37 (dear/bear mixed sausage - CA) was determined to be mixed infected with Trichinella T6 (Ct 24)/T. spiralis (Ct 37), representing a rare case of T. spiralis infection in Alaska and the first record indicating the presence of Trichinella T6 from California.

DNA sequencing analysis confirmed all single species infections identified by the Trichinella qPCR multiplex assay (including the two human biopsies). Species of the two mixed infection were confirmed by deep sequencing analysis. In both instances, amplicons of approximately $605 \mathrm{bp}$ and $490 \mathrm{bp}$ amplified using primers TCN-ITS1

C

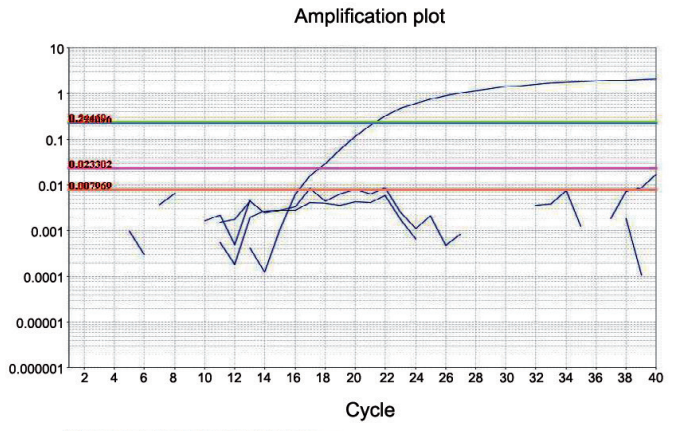

D

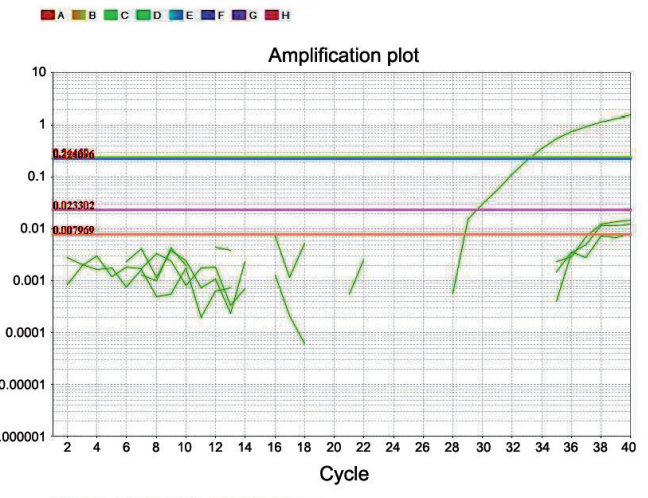

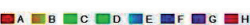

Fig. 3: amplification plots of Trichinella quantitative real-time polymerase chain reaction (qPCR) multiplex assay using T. nativa, T. spiralis, T. murrelli and Trichinella T6 specific probes. Threshold baselines are colored in blue - VIC -T. native (panel A), yellow - FAM - T. murrelli (panel B), violet - CY5 - T. spiralis (panel C) and green - NED - Trichinella T6 (panel D). 
2407F and ITS2 3020R or TCN-ITS2 3020F and 28S $3511 \mathrm{R}$, respectively were used for definitive species identification. These results are summarized in Table I.

\section{DISCUSSION}

The Trichinella qPCR multiplex assay described here was designed to target the rRNA coding region, since comparative DNA analysis performed in this study using ITS1-ITS2 region sequences revealed a significant difference among T. spiralis and T. nativa, T. murrelli and Trichinella T6. In agreement with our findings, other studies based on rRNA phylogenetic analysis of the genus Trichinella, showed similar levels of difference between these species. ${ }^{(12)}$ Compared to microscopy analysis, the assay was more sensitive for detection of clinical cases and proved to be very specific for species level differentiation of encapsulated Trichinella species found in North America.

Using our qPCR multiplex assay, 20 single Trichinella-infected and two Trichinella-mixed infected cases, i.e., T. nativa/T. spiralis and T. spiralis/Trichinella T6, were detected. The T. spiralis/Trichinella T6 mixed-infection was detected in a sausage sample (SP\#37) which was prepared with a mixture of deer and bear meat. Since deer are atypical hosts for Trichinella, ${ }^{(7)}$ and we could not identify the two types of meat in the sausage, we presumed that the infection of this sample was from the bear meat.

Specific identification of Trichinella parasites does not necessarily affect patient treatment, but it may be important as the various species have a wide range of incubation periods and clinical manifestations of the disease seem to vary by infecting species. ${ }^{(5,21)}$ Although $T$. spiralis is the most common species implicated in human infection, recently identified human cases involving $T$. murrelli and T. nativa (inclusive in this study) exemplify the complexity of the epidemiology of trichinellosis and the potential for several species of this genus to cause infections in humans. ${ }^{(22,23)}$

The assay also allowed the identification of the first confirmed case of Trichinella T6 in a bear sample from the state of California and a rare case of $T$. spiralis in Alaska. T. nativa and Trichinella T6 seem to be confined to the cold climates areas of North America, including Alaska, Colorado, Idaho, Montana, Pennsylvania and Wyoming, due their tolerance to freezing temperatures. In contrast, T. spiralis, a species relatively intolerant to freezing temperatures, seems to be restricted to southern regions. ${ }^{(9,10)}$ However, environmental adaptation of larvae is an important mechanism for dissemination of Trichinella spp., which can explain the presence of Trichinella T6 and T. spiralis in regions with adverse temperatures. ${ }^{(9,10)}$ Finally, improvements in techniques for the isolation and detection of these parasites may also explain some of the apparent expansion of Trichinella spp. into newly reported regions. ${ }^{(4)}$ Use of sensitive and specific detection methods that allow the identification of single and mixed infections is important for epidemiological studies of the geographical distribution of species in the United States.

The detection and identification of Trichinella larvae in meat samples can greatly be influenced by the larval load and by the method used for this purpose. ${ }^{(1,24)}$ The dis- crepant results observed between molecular and microscopy analysis may be partially attributable to sampling effect, as larvae are not uniformly distributed throughout a meat sample. Supporting this theory, it should be noted that $2 / 12(16 \%)$ of samples reexamined by microscopy were found to be negative on repeat microscopic analysis. This result reflects the importance of pooling different portions of the meat before performing the microscopy or PCR analysis for a conclusive diagnosis of the suspect cases. However, given the rate of detection of larval DNA in negative meat samples by microscopy, this study also highlights the relative insensitivity and lack of reproducibility of microscopy for the detection of Trichinella spp., meanly in cases when the parasite load is low.

The novel qPCR assay does not detect T. pseudospiralis, which is known to be present in North America, and has been reported from several animals, including cougars (Puma concolor cougar), ${ }^{(25)}$ Florida panthers (Puma concolor coryi) ${ }^{(26)}$ and wild boar. ${ }^{(27)}$ To date, while no human cases associated with this species have occurred on this continent, both sporadic cases of infection and outbreaks of trichinellosis due to T. pseudospiralis have occurred elsewhere. ${ }^{(28,29)}$ Unlike other Trichinella species in North America, the differentiation of this species from other trichinellae by microscopy is possible, as the larva is not encapsulated. However, in cases where infection with T. pseudospiralis is suspected and no larvae are found by microscopy, the use of $T$. pseudospiralis specific qPCR is advisable. ${ }^{(30)}$

The multiplex qPCR assay proposed here represents an advance in Trichinella spp. identification, and has an important impact on clinical cases. To our knowledge, there are no other methods published that offer the same level of specificity in a single, easy to perform assay for the identification of all encapsulated Trichinella species occurring in North America. Other protocols, designed for multi-species identification, rely on the use of multiple primers/probes, require multiple specific reactions to distinguish species, or are designed for single species identification. ${ }^{(17,18)}$

In conclusion, the molecular assay presented in this study offers improved detection and specific identification of the four encapsulated Trichinella species occurring in North America, including rare mixed infected cases. The data generated by such an assay has immediate implications for clinical epidemiological investigations and food safety, informing likely food sources for cases and indicating susceptibility of the larval Trichinella to freezing based on the species determined.

\section{ACKNOWLEDGEMENTS}

To Dr Edoardo Pozio from the European, Istituto Superiore di Sanità - Union Reference Laboratory for Parasites, Rome, Italy, for supplying the Trichinella larvae isolates used as reference controls. The authors are also indebted, to Mark Fox, Barbara Marston and Stephanie Bialek from CDC - Division of Parasitic Diseases and Malaria, Center for Global Health, USA, for invaluable assistance on preparation of illustrations and reviewing the manuscript. The findings and conclusions in this report are those of the authors and do not necessarily represent the official position of CDC. 


\section{AUTHORS' CONTRIBUTION}

MA designed primers/probes and optimized qPCR reactions, performed Sanger DNA sequencing analysis, reviewed the literature and wrote the first manuscript draft; HB and BM performed all microscopy analysis; FN performed amplicon deep sequencing and analysis; RB and AS collected the literature information and critically reviewed the manuscript. All authors read and approved the final manuscript.

\section{REFERENCES}

1. Gottstein B, Pozio E, Mockle K. Epidemiology, diagnosis, treatment, and control of trichinellosis. Clin Microbiol Rev. 2009; 22(1): $127-45$

2. Murrel KP. Worldwide occurrence and impact of human trichinellosis, 1986-2009. Emerg Infect Dis. 2011; 17(12): 2194-204.

3. Rostami AG, Gamble HR, Dupouy-Cammet J, Khanzan H, Bruschi F. Meat sources of infection for outbreaks of human trichinellosis. Food Microbiol. 2017; 64: 65-71.

4. Foreyt WJ. Trichinosis. Circular 1388. Reston: US Geological Survey; 2013. $60 \mathrm{pp}$.

5. Hall RL, Lindsey A, Hammond C, Montgomery SP, Wilkins PP, da Silva AJ, et al. Outbreak of human trichinellosis in Northern California caused by Trichinella murrelli. Am J Trop Med Hyg. 2012; 87(2): 297-302.

6. Holzbauer SM, Agger Wa, Hall RL, Johnson GM, Schmitt D, Garvey A, et al. Outbreak of Trichinella spiralis infections associated with a wild boar hunted at a game farm in Iowa. Clin Infect Dis. 2014; 59(12): 1750-6.

7. Wilson NO, Hall RL, Montgomery SP, Jones JL. Trichinellosis surveillance - United States, 2008-2012. MMWR Surveill Summ. 2015; 64(SS01): 1-8.

8. Shimoni Z, Froom P. Uncertainties in diagnosis, treatment and prevention of trichinellosis. Expert Rev Anti Infect Ther. 2015; 13(1): 1279-88.

9. Masuoka PM, Bruce R, Colaccico M, Razuri H, Hill D, Murrel KD. Predicted geographic ranges for North American sylvatic Trichinella species. J Parasitol. 2009; 95(4): 829-37.

10. Pozio E. Adaptation of Trichinella spp. for survival in cold climates. FAWPAR. 2016; 4: 4-12.

11. Pozio E, Zarlenga DS. Recent advances on the taxonomy, systematics and epidemiology of Trichinella. Int J Parasitol. 2005; 35(1112): 1191-204.

12. Pozio E, Zarlenga DS. New pieces of the Trichinella puzzle. Int J Parasitol. 2013; 43(12-13): 983-97.

13. Faber M, Shink S, Mayer-Scholl A, Ziesch C, Schonfelder R, Wichmann-SchauerH, et al. Outbreak of trichinellosis due to wild boar meat and evaluation of the effectiveness of post exposure prophylaxis, Germany, 2013. Clin Infect Dis. 2015; 60(12): e98-104.

14. Bilska-Zajac E, Rozycki M, Churzynska E, Marucci G, Cencek $\mathrm{T}$, Karamon J, et al. Trichinella species circulating in wild boar (Sus scrofa) populations in Poland. Int J Parasitol Parasites Wildl. 2013; 2: 211-3.
15. La Grange LJ, Reininghaus B, mukaratirwa S. First report of a mixed infection of Trichinella nelsoni and Trichinella T8 in a leopard (Panthera pardus) from the Greater Kruger National Park, South Africa. Onderstepoort J Vet Res. 2014; 81(1): e1-3.

16. Nockler K, Pozio E. Trichinella spiralis and Trichinella pseudospiralis mixed infection in a wild boar (Sus scrofa) of Germany. Vet Parasitol. 2006; 137(3-4): 364-8.

17. Atterby H, Learmount J, Conyers C, Zimmer I, Boonham N, Taylor M. Development of a real-time PCR assay for the detection of Trichinella spiralis in situ. Vet Parasitol. 2009; 161(1-2): 92-8.

18. Cuttell L, Corley SW, Gray CP, Vanderlinde PB, Jackson LA, Traub RJ. Real-time PCR as a surveillance tool for the detection of Trichinella infection in muscle samples from wildlife. Vet Parasitol. 2012; 188(3-4): 285-93.

19. Ash LR, Orihel T. Parasites: a guide to laboratory procedures and identification. Chicago: ASCP Press; 1987; 328 pp.

20. Zarlenga DS, Chute MB, Martin A, Kapel CM. A multiplex PCR for unequivocal differentiation of all encapsulated and non-encapsulated genotypes of Trichinella. Int J Parasitol. 1999; 29(11): 1859-67.

21. Dupouy-Camet J, Paugam A, de Pinieux G, Lavarde V, Viellefond A. Trichinella murrelli: pathological features in human muscles at different delays after infection. Parasite. 2001; 8(Suppl. 2): S176-9.

22. Khumjui C, Choomkasien P, Dekumyoy P, Kusolsuk T, Kongkaew W, Chalammat M, et al. Outbreak of trichinellosis caused by Trichinella papuae, Thailand, 2006. Emerg Infect Dis. 2008; 14(12): 1913-5.

23. Ruetsch C, Delaunay P, Armengaud A, Peloux-Petiot F, DupouyCamet $\mathrm{J}$, Vallee I, et al. Inadequate labeling of pork sausages prepared in Corsica causing a trichinellosis outbreak in France. Parasite. 2016; 23: 27.

24. Nockler K, Pozio E. Detection of Trichinella infection in food animals. Vet Parasitol. 2000; 93(3-4): 335-50.

25. Reichard MV, Logan K, Criffield M, Thomas JE, Paritte JM, Messerly DM, et al. The occurrence of Trichinella species in the cougar Puma concolor couguar from the state of Colorado and other regions of North and South America. J Helminthol. 2017; 91(3): 320-5.

26. Reichard MV, Criffield M, Thomas JE, Paritte JM, Cunningham M, Onorato D, et al. High prevalence of Trichinella pseudospiralis in Florida panthers (Puma concolor coryi). Parasit Vectors. 2015; 8: 67.

27. Gamble HR, Pozio E, Lichtenfels JR, Zarlenga DS, Hill DE. Trichinella pseudospiralis from a wild pig in Texas. Vet Parasitol. 2005; 132(1-2): 147-50.

28. Jongwutiwes $S$, Chantachum N, Kraivichian P, Siriyasaties $P$, Putaporntip C, Tamburrini A, et al. First outbreak of human trichinellosis caused by Trichinella pseudospiralis. Clin Infect Dis. 1998; 26(1): 111-5.

29. Ranque S, Faugere B, Pozio E, La Rosa G, Tamburrini A, Pillisier JF, et al. Trichinella pseudospiralis outbreak in France. Emerg Infect Dis. 2000; 6(5): 543-7.

30. Guenther S, Nockler K, von Nickisch-Rosenegk M, Landgraf M, Ewers C, Wieler LH, et al. Detection of Trichinella spiralis, $T$. britovi and T. pseudospiralis in muscle tissue with real-time PCR. J Microbiol Methods. 2008; 75(2): 287-92. 